\title{
CONDIÇÃO BUCAL E ESTADO NUTRICIONAL DE PACIENTES DE CLÍNICAS ODONTOLÓGICAS DE CIDADES LITORÂNEAS DO ESTADO DE SÃO PAULO
}

\section{ORAL CONDITION AND NUTRITIONAL STATUS OF PATIENTS IN DENTAL CLINICS OF COASTAL CITIES IN THE STATE OF SÃO PAULO}

\author{
Daniele Lima da Cruz' \\ Aline Veroneze de Mello ${ }^{1}$ \\ Luana Romão Nogueira ${ }^{1}$ \\ Walleska Luctke Villarim ${ }^{1}$ \\ Vanessa Apollinario' \\ Rosana Farah Simony Lamigueiro Toimil ${ }^{2}$
}

Resumo: A saúde bucal é indicativa de diversas enfermidades e pode afetar o estado nutricional. O objetivo do presente estudo foi relacionar a saúde bucal com o estado nutricional de pacientes de clínicas odontológicas do litoral de São Paulo. Estudo transversal com pacientes de clínicas odontológicas, com idade a partir de 20 anos por intermédio de questionário, com perguntas para caracterização da amostra, classificação do estado nutricional, periodicidade de visitas ao dentista, condição bucal e consumo alimentar. Participaram 53 pacientes, sendo 71,7\% mulheres e $28,3 \%$ homens, com média de idade de 32,6 25,46 anos e $83 \%$ possuíam ensino médio completo. $A$ maioria (54,72\%) encontra-se na faixa de eutrofia. Sobre a relação do estado nutricional com a saúde bucal, $22,64 \%$ dos eutróficos utilizavam aparelho ortodôntico fixo. Edentulismo e prótese total representam 5,66\% dos problemas bucais em eutróficos, 58,49\% ingerem líquidos durante as refeições. Os pacientes preferem: alimentos consistentes, 32,08\%; picados, 22,64\%; sopas, 20,75\%; amassados, 11,32\%; de qualquer consistência, 11,32\%; e 1,89\%, moídos. Entre os usuários de aparelho ortodôntico, $15 \%$ preferem carnes. Indivíduos com cárie não consomem ou evitam: legumes e vegetais crus e doces (87,5\%), carnes e queijos (75\%). Usuários de prótese total ou parcial optam por frutas picadas e carnes picadas e macias (75\%), legumes e vegetais cozidos (62,5\%), evitam ou não consomem frutas inteiras e carnes (87,5\%); e legumes e vegetais crus (75\%). Embora a condição bucal da maioria dos pacientes apresente algum fator a ser considerado, a maioria dos indivíduos encontra-se em eutrofia, indicando que, na população estudada, não há uma relação direta entre o estado nutricional e sua condição bucal. Palavras-chave: estado nutricional; saúde bucal; preferências alimentares.

Abstract: Oral health is an important indication of various diseases and can affect nutritional status. The objective of this study is to relate oral health with nutritional status of patients from dental clinics on the coast of São Paulo. This is a Cross-sectional study with patients of dental clinics, who were 20 years old or greater. A questionnaire was prepared, containing questions for sample characterization, rating of nutritional status, frequency of dental appointments, oral health status, and dietary intake. Fifty-three patients participated, $71.7 \%$ were women and $28.3 \%$ were men, the average age was about $32.6 \pm 25.46$ years old and $83 \%$ of the subjects had completed the high school. The majority (54.72\%) were of normal weight. Regarding the relation between nutritional status and oral health, 22.64\% of normal weight individuals were wearing braces. Edentulism and complete denture represent $5.66 \%$ of oral health problems in normal weight subjects, whereas $58.49 \%$ ingest liquids during meals. Patient's preferences: $32.08 \%$ solid food, $22.64 \%$ chopped food, $20.75 \%$ soups, $11.32 \%$ prefer mashed food, $11.32 \%$ any

\footnotetext{
1 Graduanda do curso de Nutrição - Universidade Presbiteriana Mackenzie - UPM. E-mails: danny_Ic@msn.com; alinevm3@hotmail.com; luanaromaon@hotmail.com; walleskao@gmail.com; vanapollinario@gmail.com.

${ }^{2}$ Doutora em Ciências Médicas - Universidade Federal de São Paulo - Unifesp. Docente do curso de Nutrição Universidade Presbiteriana Mackenzie - UPM. E-mail: rosanafarah@gmail.com.
} 
consistency, and $1.89 \%$ milled food. Among users of braces, $15 \%$ prefer meat. Individuals with caries did not consume or avoided raw vegetables and sweets (87.5\%) and whole meats and cheeses (75\%). Users of complete or partial denture opted for chopped fruits, soft and portioned meat (75\%), boiled vegetables (62,5\%), and avoided or did not consume whole fruits and meats (87.5\%) and raw vegetables (75\%). It is important to maintain regular visits to the dentist to detect problems with oral health. Then there are not changes in dietary habits and preferences. Keywords: nutritional status; oral health; food preferences.

\section{INTRODUÇÃO}

A saúde bucal é parte integrante e inseparável da saúde geral e que pode afetar o bemestar físico e psicossocial das pessoas. Está relacionada às condições socioeconômicas, alimentares, educacionais e de acesso aos serviços de saúde e à informação (WORLD HEALTH ORGANIZATION, 2004). A boca é um indicador precoce da saúde geral e do estado nutricional. Sintomas e sinais clínicos relacionados à nutrição e outros problemas de saúde frequentemente surgem, primeiro, na cavidade oral (PALMER et al., 2010).

A prestação de serviços públicos de saúde bucal no Brasil caracterizava-se por ações de baixa complexidade e a grande maioria dos municípios brasileiros desenvolvia ações para a faixa etária escolar ( 6 a 12 anos) e gestantes, o que teve impacto positivo na saúde bucal dessa população (BRASIL, 2003). Porém, adultos e idosos tinham somente o acesso aos serviços de pronto atendimento e de urgência. Dessa forma, em 2003, foi realizado pelo Ministério da Saúde o primeiro Levantamento Nacional de Saúde Bucal (SB Brasil), no qual indicou que $13 \%$ dos adolescentes nunca haviam ido ao dentista, $20 \%$ da população brasileira já tinha perdido todos os dentes e $45 \%$ dos brasileiros não possuíam acesso regular à escova de dentes. Desde então, foi implementado o Programa Brasil Sorridente, que integra a Política Nacional de Saúde Bucal do Sistema Único de Saúde (SUS), tendo por objetivo aumentar os procedimentos odontológicos à população brasileira (BRASIL, 2013).

Atualmente, apesar dos avanços relacionados ao cuidado com a saúde bucal, as perdas dentárias decorrentes de cáries e de doença periodontais ainda são prevalentes. O SUS não tem estrutura suficiente para absorver a demanda por atenção à saúde bucal da população adulta. Por isso, dentes que poderiam ser recuperados são extraídos, já que essa é uma alternativa considerada mais prática e também mais econômica (SILVA et al., 2010).

Os indivíduos que apresentam ausência total ou parcial de dentes têm problemas em funções importantes, tais como a fala e mastigação, o que, consequentemente, acarreta prejuízos na comunicação e nutrição. Esses prejuízos podem levar à diminuição da autoestima, dificuldades de socialização, sensação de envelhecimento e sentimento de humilhação (SILVA; MAGALHÃES; FERREIRA, 2010). Ter a função mastigatória reduzida por perdas dentárias, utilização de próteses, presença de cáries ou doenças periodontais, lesão de tecidos moles e disfunções temporomandibulares - DTM (BRAGA, 2002), podem aumentar os riscos de câncer, doenças cardiovasculares, além de problemas gastrointestinais. Para avaliar problemas decorrentes da diminuição da eficiência mastigatória, podem ser aplicados testes para medir força muscular, bem como questionários sobre hábitos alimentares 
(ANDRADE; SEIXAS, 2006; BERNARDO et al., 2012).

A relação existente entre saúde dental e nutrição ocorre devido ao fato dos indivíduos afetados acabarem consumindo menos frutas, vegetais, legumes e fibras, comprometendo, dessa forma, a saúde e a qualidade de vida e podendo ser fator de risco para diversas doenças crônicas (DE MARCHI, 2008).

O alimento, uma vez na cavidade oral, pode influenciar a formação e o metabolismo de placas bacterianas de acordo com sua composição química e características físicas. Já, os nutrientes agem na formação e crescimento dos dentes, o que vai determinar a resposta à ação dessas placas. Mudanças no padrão alimentar da população, como a substituição de alimentos ricos em fibras e nutrientes por alimentos industrializados, ricos em gorduras e carboidratos, favorecem o surgimento de doenças odontológicas (CRISPIM et al., 2010).

Alguns fatores podem contribuir para a melhora da saúde bucal, como manter uma alimentação equilibrada, higiene bucal, visitas regulares ao dentista, além de um estado nutricional adequado (CAMPOS; FOSCHINI, 2012). Faz-se necessária a inclusão de orientações nutricionais no atendimento a pacientes em tratamento odontológico com o auxílio de nutricionista (OLIVEIRA JR et al., 2008). Os Guias Alimentares podem ser utilizados para direcionar a população a um modo de vida mais saudável, pois fatores de risco para doenças crônicas não-transmissíveis (DCNTs) também são determinantes para o surgimento de doenças bucais. Logo, as informações contidas nesses documentos podem, também, ser utilizadas na elaboração e monitoramento de políticas de saúde pública visando à saúde bucal da população (FREIRE et al., 2012).

Há escassez de dados na literatura que correlaciona a saúde bucal com a alimentação e o estado nutricional da amostra; portanto, este estudo tem como finalidade relacionar a saúde bucal com o estado nutricional de pacientes de algumas clínicas odontológicas de cidades litorâneas do estado de São Paulo. Entre os objetivos específicos estão: coletar dados de saúde bucal desses pacientes; verificar se o consumo alimentar é alterado devido à saúde bucal e classificar o estado nutricional dos pacientes e relacionar a saúde bucal à alimentação.

\section{METODOLOGIA}

Foi realizado estudo transversal, entre os meses de agosto e novembro de 2013, no qual foram entrevistados pacientes de clínicas odontológicas, com idade a partir de 20 anos, localizadas em cidades litorâneas do estado de São Paulo (Santos, Cubatão e Guarujá).

Foi elaborado um questionário especificamente para o presente estudo, contendo dados antropométricos, nutricionais, habituais e odontológicos. Para a caracterização da amostra: nome, idade, sexo, escolaridade. Os dados antropométricos avaliados, tais como o Peso $(\mathrm{kg})$ e Altura $(m)$, foram autorreferidos pelo entrevistado, para cálculo de Índice de Massa Corporal (IMC), por meio da fórmula P/A² e classificação do estado nutricional de acordo com os pontos de corte preconizados pela Organização Mundial da Saúde - OMS (2002), ou seja, baixo peso 
(IMC < 18,5); eutrofia, sendo definida por indivíduos que possuem estado nutricional adequado (IMC 18,5-24,99); sobrepeso (IMC 25-29,99) e obesidade (IMC $\geq 30,00$ ), para indivíduos com até 60 anos. Para pessoas com mais de 60 anos, o estado nutricional foi classificado de acordo com a Organização Pan-Americana de Saúde (2002).

As questões odontológicas abrangiam a periodicidade de visitas ao dentista (semanal, quinzenal, mensal, semestral e anual), problema odontológico apresentado (cáries, disfunção temporomandibular, edentulismo, prótese parcial, prótese total, implantes, aparelho ortodôntico, dificuldade ou dor ao mastigar, dificuldade ou dor ao engolir) e quantidade de dentes naturais, foram respondidas pelo dentista e as questões referentes à ingestão de líquido durante as refeições para auxiliar na deglutição e consistência do alimento foram respondidas, com um "X”, pelo próprio entrevistado.

A questão nutricional referente ao consumo alimentar foi respondida com "2" para os frequentemente consumidos, "1" para os consumidos ocasionalmente e "0" para os não consumidos, sendo opções: carnes inteiras, picadas ou macias, legumes e vegetais crus ou cozidos, leite, queijos, ovos, pães macios, torradas, biscoitos, frutas inteiras ou picadas e macias e doces.

Dessa maneira, todos os participantes receberam Termo de Esclarecimento Livre e Esclarecido (TCLE), contendo informações referentes aos procedimentos que foram realizados na pesquisa. As instituições participantes do estudo receberam carta de informação e TCLE, concordando participar do estudo. A pesquisa atendeu aos princípios éticos da Resolução do CNS 196/96 e foi aprovada pelo CIEP nº N001/02/11.

Foram incluídos, no estudo, frequentadores de clínicas odontológicas, adultos e idosos das seguintes cidades litorâneas: Santos (15 indivíduos), Cubatão (13 indivíduos) e Guarujá (25 indivíduos); e, também, do município de São Paulo. A pesquisa contou com um número amostral inicial de 60 participantes, porém como o número de pessoas do município de São Paulo foi pequeno, não foi possível realizar o estudo comparativo entre as cidades litorâneas e esse município. Dessa forma, foram excluídos os residentes do município, bem como indivíduos adolescentes.

\section{RESULTADOS E DISCUSSÃO}

O presente estudo contou com uma amostra inicial de 60 participantes, mas somente 53 pacientes adultos e idosos de ambos os gêneros que frequentam clínicas odontológicas de cidades litorâneas do estado de São Paulo foram avaliados, sendo $71,7 \%$ do gênero feminino $(n=38)$ e $28,3 \%$ do gênero masculino $(n=15)$. A média de idade foi de $32,6 \pm 25,46$ anos, variando entre 20 e 80 anos. Quanto à escolaridade, 83\% possuíam ensino médio completo, $32 \%$ ensino superior completo, e 9,43\% eram pós-graduados.

Quanto à quantidade de dentes, a média foi de $26,15 \pm 8,34$. Resultado ligeiramente

acima do encontrado no estudo de Figueiredo et al. (2011), que descreveram a saúde bucal 
e o perfil socioeconômico de uma população adulta (18 a 60 anos), residente no Rio Grande do Sul, onde a média de dentes perdidos, por pessoa, na população, em geral, é de 9. Perdas dentárias acima de 10 elementos já causam prejuízos à funcionalidade da mastigação e na fala, afetando diretamente a qualidade de vida e a autoestima do indivíduo (FIGUEIREDO et al., 2011).

Quanto ao estado nutricional, conforme Tabela 1, a maioria dos pesquisados $(54,72 \%)$ encontra-se na faixa de eutrofia, seguidos pelos indivíduos em sobrepeso (32,08\%). Resultado que diverge do encontrado por Sheiham et al. (2002), que tinham como objetivo avaliar a relação entre saúde bucal e IMC. Nesse trabalho, a maioria dos indivíduos estava na faixa de sobrepeso $(47,4 \%)$, seguido pelos eutróficos $(29,9 \%)$. Esses autores afirmam que indivíduos com menos de 21 dentes têm maior probabilidade de terem sobrepeso e obesidade do que indivíduos com mais de 21 dentes. As divergências nos resultados do presente estudo com o trabalho consultado podem ser explicadas pela diferença de faixa etária, nacionalidade da população estudada e quantidade de dentes da amostra.

Tabela 1 - Distribuição, em porcentagem, dos pacientes de clínicas odontológicas de acordo com estado nutricional, São Paulo, 2013

\begin{tabular}{ccc}
\hline Estado Nutricional & $\mathbf{n}$ & $\%$ \\
\hline Desnutrição & 1 & 1,89 \\
Eutrofia & 29 & 54,72 \\
Sobrepeso & 17 & 32,08 \\
Obesidade grau I & 4 & 7,55 \\
Obesidade grau II & 2 & 3,77 \\
Total & 53 & 100,00 \\
\hline
\end{tabular}

Sobre a relação do estado nutricional com a saúde bucal, presente no Quadro 1, o item mais comum foi o uso de aparelho ortodôntico fixo para os indivíduos na faixa de eutrofia $(22,64 \%)$ e em indivíduos com sobrepeso $(9,43 \%)$. Edentulismo e prótese total representam $5,66 \%$ dos problemas bucais em eutróficos. Devido a uma quantidade significativa dos indivíduos, da presente pesquisa, estar na faixa de eutrofia, a maior parte dos problemas bucais estão concentrados nessa faixa populacional. Nas demais classificações de estado nutricional, a cárie dentária (7,55\% em sobrepeso) é o problema mais comum.

O uso de aparelhos ortodônticos pode acarretar alterações na mastigação, deglutição e fala (NAVARRO et al., 2012). O edentulismo (ausência de dentes) influencia na mastigação, o que reflete no paladar, pronúncia, e estética, além da digestão. Leva a mudanças nas escolhas alimentares e preparo dos alimentos. $O$ uso de próteses totais pode auxiliar na recuperação da função mastigatória para indivíduos que perderam os dentes. No entanto, pessoas com próteses mastigam de 75 a $80 \%$ menos eficientemente que aquelas com dentes naturais, o que pode causar uma diminuição na ingestão de carnes, frutas e vegetais frescos e aumento de consumo de alimentos mais macios (SILVA, 2011). 
Quadro 1 - Distribuição, em porcentagem, do estado nutricional de pacientes de clínicas odontológicas de acordo com condição bucal, São Paulo, 2013

\begin{tabular}{|c|c|c|c|c|c|c|c|c|}
\hline \multirow{3}{*}{ Doença Bucal } & \multicolumn{8}{|c|}{ Estado Nutricional } \\
\hline & \multicolumn{2}{|c|}{ Desnutrição } & \multicolumn{2}{|c|}{ Eutrofia } & \multicolumn{2}{|c|}{ Sobrepeso } & \multicolumn{2}{|c|}{$\begin{array}{c}\text { Obesidade grau I } \\
\text { e II }\end{array}$} \\
\hline & $n$ & $\%$ & $\mathbf{n}$ & $\%$ & $\mathbf{N}$ & $\%$ & $\mathbf{n}$ & $\%$ \\
\hline Cárie & 1 & 1,89 & 2 & 3,77 & 4 & 7,55 & 1 & 1,89 \\
\hline Disfunção temporomandibular & 0 & 0,00 & 0 & 0,00 & 1 & 1,89 & 0 & 0,00 \\
\hline Edentulismo & 0 & 0,00 & 3 & 5,66 & 1 & 1,89 & 0 & 0,00 \\
\hline Prótese Parcial & 0 & 0,00 & 1 & 1,89 & 1 & 1,89 & 2 & 3,77 \\
\hline Prótese Total & 0 & 0,00 & 3 & 5,66 & 1 & 1,89 & 0 & 0,00 \\
\hline Implantes & 0 & 0,00 & 2 & 3,77 & 1 & 1,89 & 0 & 0,00 \\
\hline Aparelho Ortodôntico & 1 & 1,89 & 12 & 22,64 & 5 & 9,43 & 2 & 3,77 \\
\hline Dificuldade ou dor ao mastigar & 0 & 0,00 & 1 & 1,89 & 1 & 1,89 & 0 & 0,00 \\
\hline Disfagia & 0 & 0,00 & 0 & 0,00 & 0 & 0,00 & 1 & 0,00 \\
\hline
\end{tabular}

Referente à média de visitas ao dentista (Figura 1), grande parte dos pesquisados $(35,85 \%)$ realiza visitas mensais a esse profissional, seguido de $32,08 \%$ que comparecem a clinicas odontológicas semestralmente. Resultado próximo ao encontrado por Rios e Costa (2009), que tinham como objetivo avaliar o impacto da periodicidade autorreportada de visitas ao dentista sobre parâmetros clínicos periodontais. Os autores concluíram que $43,3 \%$ de sua amostra comparecem a consultas odontológicas mais de uma vez por ano. Visitas regulares ao dentista estão associadas a melhoras em parâmetros periodontais e a uma melhor condição bucal.

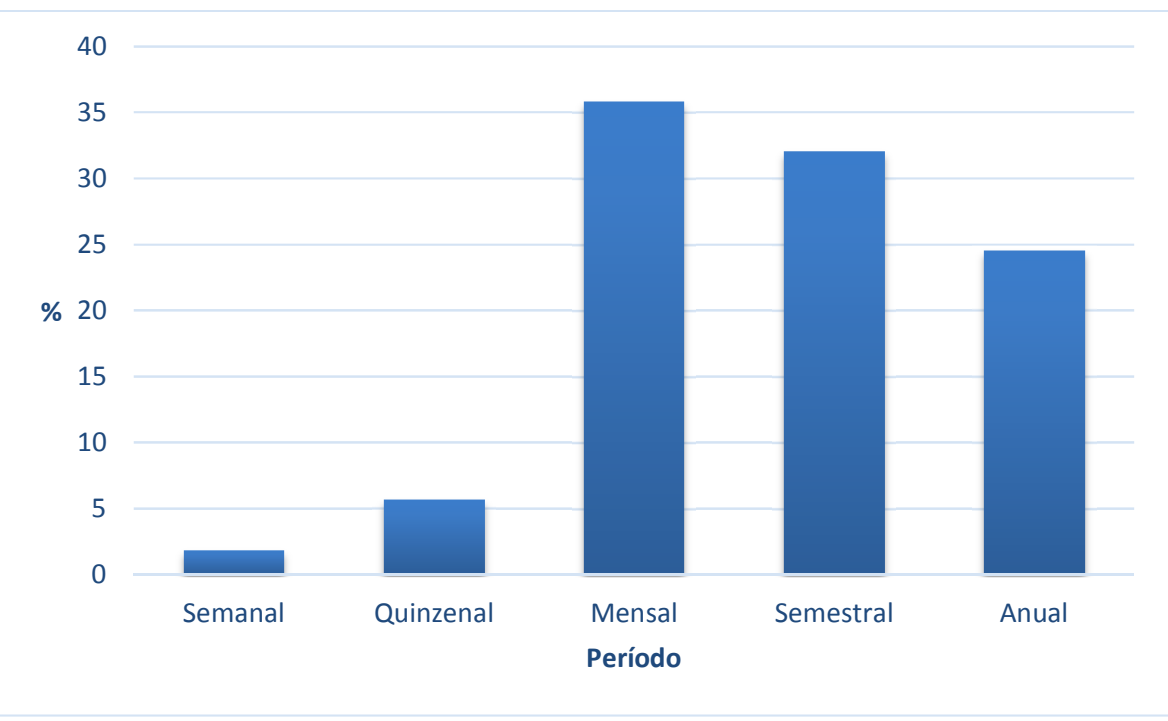

Figura 1 - Distribuição, em porcentagem, da frequência de vistas de pacientes de clínicas odontológicas. São Paulo, 2013. 
Quanto ao hábito de ingerir líquidos durante as refeições, para auxiliar na deglutição, $58,49 \%$ dos indivíduos afirmaram ter esse hábito e $41,51 \%$ afirmaram não ter essa necessidade. Resultado semelhante encontrado por Jorge et al. (2009), que tinham como objetivo investigar se as perdas dentárias em indivíduos adultos apresentam relação com as queixas de fala, mastigação e deglutição. De acordo com esses autores, alterações na deglutição podem ser decorrentes de problemas na mastigação, uma vez que, ao deglutir partículas grandes e pouco umedecidas, o indivíduo apresenta maior esforço, alterando a postura de cabeça e a ação da musculatura envolvida.

No presente estudo, uma grande porcentagem dos pacientes têm como forma de preferência de consumo: alimentos consistentes, 32,08\%; picados, $22,64 \%$; sopas, 20,75\%; amassados, 11,32\%; de qualquer consistência, 11,32\%; e apenas 1,89\%, moídos. Em estudo realizado com 50 pacientes, de ambos os sexos, com idades variando entre 33 e 76 anos, atendidos na Clínica de Prótese Dentária do Departamento de Prótese e Cirurgia Buco Facial da Universidade Federal de Pernambuco (ANDRADE; SEIXAS, 2006), foram obtidos resultados divergentes, sendo que $40 \%$ preferiam os alimentos picados; $24 \%$ preferiam consistentes; $24 \%$, amassados; $8 \%$, moídos; e $4 \%$, sopas e liquidificados, fato que pode ser explicado por se tratarem de indivíduos usuários de próteses totais que apresentam certa dificuldade de mastigação.

Quanto ao tipo de alimento consumo, entre os três problemas bucais mais comuns no presente estudo: aparelho ortodôntico, cárie e próteses totais ou parciais, são mostrados no Quadro 2.

\section{Quadro 2 - Distribuição em porcentagem dos grupos de alimentos mais consumidos e dos não consumidos e evitados segundo a condição bucal, São Paulo, 2013}

\begin{tabular}{|c|c|c|c|c|c|c|}
\hline \multirow{2}{*}{ Grupo de alimentos } & \multicolumn{2}{|c|}{$\begin{array}{c}\text { Aparelho } \\
\text { Ortodôntico (n=20) }\end{array}$} & \multicolumn{2}{c|}{ Cáries (n=8) } & \multicolumn{2}{c|}{$\begin{array}{c}\text { Próteses totais ou } \\
\text { parciais (n=8) }\end{array}$} \\
\hline & $\begin{array}{c}\text { Mais } \\
\text { consu- } \\
\text { midos }\end{array}$ & $\begin{array}{c}\text { Não } \\
\text { consumi- } \\
\text { dos ou } \\
\text { evitados }\end{array}$ & $\begin{array}{c}\text { Mais } \\
\text { consu- } \\
\text { midos }\end{array}$ & $\begin{array}{c}\text { Não } \\
\text { consumi- } \\
\text { dos ou } \\
\text { evitados }\end{array}$ & $\begin{array}{c}\text { Mais } \\
\text { consu- } \\
\text { midos }\end{array}$ & $\begin{array}{c}\text { Não } \\
\text { consumi- } \\
\text { dos ou } \\
\text { evitados }\end{array}$ \\
\cline { 2 - 8 } & \multicolumn{2}{|c|}{$\%$} & \multicolumn{2}{c|}{$\%$} \\
\hline Carnes Inteiras & 15 & 85 & 25 & 75 & 12,5 & 87,5 \\
\hline Carnes Picadas & 75 & 25 & 75 & 25 & 75 & 25 \\
\hline Legumes e vegetais crus & 20 & 80 & 12,5 & 87,5 & 25 & 75 \\
\hline Legumes e vegetais cozidos & 50 & 50 & 62,5 & 37,5 & 62,5 & 37,5 \\
\hline Leite & 70 & 30 & 37,5 & 62,5 & 75 & 25 \\
\hline Queijos & 35 & 65 & 25 & 75 & 75 & 25 \\
\hline Ovos & 50 & 50 & 50 & 50 & 62,5 & 37,5 \\
\hline Pães moles & 75 & 25 & 62,5 & 37,5 & 37,5 & 12,5 \\
\hline Torradas, biscoitos, bolachas & 45 & 55 & 37,5 & 62,5 & 87,5 & 12,5 \\
\hline Frutas inteiras & 25 & 75 & 37,5 & 62,5 & 12,5 & 87,5 \\
\hline Frutas macias & 45 & 55 & 62,5 & 37,5 & 75 & 25 \\
\hline Doces & 35 & 65 & 12,5 & 87,5 & 37,5 & 62,5 \\
\hline
\end{tabular}


Entre os usuários de aparelho ortodôntico, observa-se que o consumo de carnes inteiras foi relatado apenas por $15 \%$. Em pesquisa realizada pelo Hospital Universitário $(\mathrm{HU})$ da Universidade Federal do Maranhão com 50 pacientes, de ambos os sexos, na faixa etária de 20 a 39, observou-se que a maior diminuição no nível de consumo foi no grupo das carnes (44\% da amostra), após a colocação do aparelho, o que é explicado pelo fato de ser um alimento que necessita de certa força muscular e maior número de mastigações (OLIVEIRA JUNIOR et al., 2008). Além disso, no presente estudo, poucos consomem frutas inteiras (25\%) e legumes e vegetais crus $(20 \%)$, pois possuem a necessidade de mastigação e força muscular semelhante ao das carnes.

Já os indivíduos que relataram possuírem cárie têm como alimentos não consumidos ou evitados: legumes e vegetais crus e doces $(87,5 \%)$, carnes inteiras e queijos $(75 \%)$. As cáries são ocasionadas pela presença de carboidratos fermentáveis e de sacarose na cavidade oral, e podem provocar dores (DIAS; RASLAN; SCHERMA, 2011). Dessa maneira, isso pode explicar a restrição desses grupos de alimentos (doces e queijos; carnes inteiras e vegetais crus, respectivamente).

Quanto à preferência alimentar dos usuários de prótese total ou parcial $(n=8)$, pode-se perceber que os pacientes optam por frutas picadas e carnes picadas e macias $(75 \%)$, legumes e vegetais cozidos $(62,5 \%)$ e evitam ou não consomem frutas inteiras e carnes inteiras (87,5\%) e legumes e vegetais crus (75\%). Em estudo realizado por Lima et al. (2007), com 58 participantes idosos, usuários de próteses totais, atendidos no Complexo Odontológico de Cruz das Armas (COCA), da cidade de João Pessoa, foi constatado que os alimentos que representam os mais evitados foram carnes $(22,45 \%)$, saladas e vegetais crus $(15,5 \%)$ e frutas $(13,8 \%)$.

\section{CONCLUSÃO}

Um fator limitante deste estudo foi o fato de alguns dados serem secundários (colhidos de prontuários). Ainda assim, pode-se perceber que, embora a condição bucal da maioria dos pacientes apresente algum fator a ser considerado, a maioria dos indivíduos encontra-se em eutrofia, indicando que, na população estudada, não há uma relação direta entre o estado nutricional e a sua condição bucal. Por outro lado, ao realizar a análise das preferências alimentares entre pacientes com aparelho ortodôntico, cárie e próteses totais ou parciais, verificou-se que apresentam alterações no hábito alimentar, principalmente com relação à diminuição do consumo de carnes, legumes e vegetais crus, frutas inteiras. É importante manter visitas regulares ao dentista para se detectar, precocemente, problemas com a saúde bucal para que não haja alterações nos hábitos e preferências alimentares decorrentes desses problemas. 


\section{REFERÊNCIAS BIBLIOGRÁFICAS}

ANDRADE, B. M. S.; SEIXAS, Z. A. Condição mastigatória de usuários de próteses totais. International Journal of Dentistry, Recife, v. 1, n. 2, p. 48-51, jun. 2006.

BERNARDO, C. O. et al. Association between tooth loss and obesity in Brazilian adults: a population based study. Revista de Saúde Pública, São Paulo, v. 46, n. 5, p. 834-842, out. 2012.

BRAGA, S. R. S. et al. Efeito do uso de próteses na alimentação de idosos. Revista de Odontologia UNESP, Marília, v. 31, n. 1, p. 71-81, jan./jul. 2002.

BRASIL. Ministério da Saúde. Brasil Sorridente. 2013. Disponível em: <http://dab.saude.gov.br/portaldab/ape_brasil_sorridente.php>. Acesso em: 28 ago. 2013.

CAMPOS, J. A. D. B.; FOSCHINI, A. L. R. Associação da cárie dentária com indicadores antropométricos do estado nutricional em pré-escolares. Alimentos e Nutrição, Araraquara, v. 23, n. 4, p. 597-602, out./dez. 2012.

CRISPIM, M. G. A. et al. Saúde bucal e sua associação com o estado nutricional e a condição socioeconômica em adolescentes. Revista Gaúcha de Odontologia, Porto Alegre, v. 58, n. 1, p. 41-46, jan./mar. 2010.

DE MARCHI, R. J. et al. Association between oral health status and nutritional status in south Brazilian independent-living older people. Nutrition, v. 24, n. 6, p. 546-553, jun. 2008.

DIAS, A. C. G.; RASLAN, S., SCHERMA, A. P. Aspectos nutricionais relacionados à prevenção de cáries na infância. Cli. Pesq. Odonto UNITAU, Taubaté, v. 3, n. 1, p. 37-44, 2011.

FIGUEIREDO, M. C. et al. Descrição da saúde bucal e de indicadores socioeconômicos de uma população adulta. Publ. UEPG Ciência, Biologia e Saúde, Ponta Grossa, v. 17, n. 2, p. 83-89, jul./dez. 2011.

FREIRE, M. C. M. et al. Guias alimentares para a população brasileira: implicações para a Política Nacional de Saúde Bucal. Cad. de Saúde Pública, Rio de Janeiro, v. 28, Supl. S20S29, 2012.

JORGE, T. M. et al. Relação entre perdas dentárias e queixas de mastigação, deglutição e fala em indivíduos adultos. Revista CEFAC, Rio de Janeiro, v. 11, Supl. 3, p. 391-397, 2009.

LIMA, L. H. M. A. et al. Autopercepção oral e seleção de alimentos por idosos usuários de próteses totais. Revista de Odontologia da UNESP, Araçatuba, v. 36, n. 2, p. 131-136, 2007.

NAVARRO, P. R. et al. Alterações de funções orais na presença de aparelhos ortodônticos fixos com recursos intraorais. Revista CEFAC, Rio de Janeiro, v. 15, n. 5, p. 1281-1291, 2012.

OLIVEIRA JUNIOR, J. D. A. et al. Avaliação dos hábitos de pacientes adultos submetidos ao tratamento ortodôntico. Pesquisa Brasileira Odontopediatria e Clínica Integrada, João 
Pessoa, v. 8, n. 3, p. 283-288, set./dez. 2008.

ORGANIZAÇÃO PAN-AMERICANA DE SAUDE (OPAS). Encuestra Multicêntrica - Salud Beinestar $Y$ Envejecimeiento (SABE) en América Latina e el Caribe: Informe preliminar. In: REUNIÓN DEL COMITÊ ASESOR DE IVESTIGACIONES EN SALUD, 36., 2001, Jamaica. Anais... Jamaica: División de Promoción y Protección de la Salud, 2001, ABNE; 2012.

PALMER, C. A. et al. Important Relationships Between Nutrition and oral health. Nutrition Today, Baltimore, v. 45, n. 4, p. 154-164, 2010.

RIOS, F. S.; COSTA, R. S. A. Visitas ao dentista auto-reportadas e condição periodontal: um estudo de Porto Alegre. Porto Alegre. 2009. 29p. Graduação (Conclusão de Curso de Odontologia) - Universidade Federal do Rio Grande do Sul, Porto Alegre, 2009.

SHEIHAM, A. et al. The relationship between oral health status and Body Mass Index among older people: a national survey of older people in Great Britain. British Dental Journal, Londres, v. 192, n. 12, p. 703-706, jun. 2002.

SILVA, M. E. S; MAGALHÃES, C. S.; FERREIRA, E. F. Perda dentária e expectativa da reposição protética: estudo qualitativo. Ciência \& Saúde Coletiva, Rio de Janeiro, v. 15, n. 3 , p. 813-820, maio 2010.

SILVA, M. E. S. et al. Impacto da perda dentária na qualidade de vida. Ciência e Saúde Coletiva, Rio de Janeiro, v. 15, n. 3, p. 841-850, maio 2010.

SILVA, L. T. Alterações bucais do envelhecimento e implicações para a atenção odontológica. Conselheiro Lafaiete. 2011. 42f. Especialização (Conclusão de curso) Universidade Federal de Minas Gerais, Conselheiro Lafaiete, 2011.

WORLD HEALTH ORGANIZATION (WHO). Effective use of fluorides for the prevention of dental caries in the 21 st century: the WHO approach. CommunityDent Oral Epidemiology, v. 32, p. 319-321, 2004. 